\title{
Computational identification of variables in neonatal vocalizations predictive for postpubertal social behaviors in a mouse model of $16 \mathrm{p} 11.2$ deletion
}

\author{
Mitsuteru Nakamura $\mathbb{1}^{1,2} \cdot$ Kenny $\mathrm{Ye}^{3} \cdot$ Mariel Barbachan e Silva ${ }^{4} \cdot$ Takahira Yamauchi $^{1} \cdot$ Daniel J. Hoeppner $\mathbb{D}^{5}$. \\ Amir Fayyazuddin $\mathbb{1}^{5} \cdot$ Gina Kang $^{1} \cdot$ Emi A. Yuda $^{6} \cdot$ Masako Nagashima $^{7}$. Shingo Enomoto ${ }^{7}$. Takeshi Hiramoto ${ }^{1}$. \\ Richard Sharp ${ }^{8} \cdot$ Itaru Kaneko $\mathbb{1}^{9} \cdot$ Katsunori Tajinda $^{5} \cdot$ Megumi Adachi $^{5} \cdot$ Takuma Mihara $^{10} \cdot$ Shinichi Tokuno $^{2,11}$. \\ Mark A. Geyer ${ }^{8}$ Pilib Ó Broin ${ }^{4} \cdot$ Mitsuyuki Matsumoto $^{5,10} \cdot$ Noboru Hiroi $\mathbb{B}^{1,12,13,14}$
}

Received: 15 November 2020 / Revised: 12 March 2021 / Accepted: 29 March 2021 / Published online: 15 April 2021

(c) The Author(s) 2021. This article is published with open access

\begin{abstract}
Autism spectrum disorder (ASD) is often signaled by atypical cries during infancy. Copy number variants (CNVs) provide genetically identifiable cases of ASD, but how early atypical cries predict a later onset of ASD among CNV carriers is not understood in humans. Genetic mouse models of CNVs have provided a reliable tool to experimentally isolate the impact of CNVs and identify early predictors for later abnormalities in behaviors relevant to ASD. However, many technical issues have confounded the phenotypic characterization of such mouse models, including systematically biased genetic backgrounds and weak or absent behavioral phenotypes. To address these issues, we developed a coisogenic mouse model of human proximal 16p11.2 hemizygous deletion and applied computational approaches to identify hidden variables within neonatal vocalizations that have predictive power for postpubertal dimensions relevant to ASD. After variables of neonatal vocalizations were selected by least absolute shrinkage and selection operator (Lasso), random forest, and Markov model, regression models were constructed to predict postpubertal dimensions relevant to ASD. While the average scores of many standard behavioral assays designed to model dimensions did not differentiate a model of 16p11.2 hemizygous deletion and wild-type littermates, specific call types and call sequences of neonatal vocalizations predicted individual variability of postpubertal reciprocal social interaction and olfactory responses to a social cue in a genotype-specific manner. Deepphenotyping and computational analyses identified hidden variables within neonatal social communication that are predictive of postpubertal behaviors.
\end{abstract}

\section{Introduction}

Because copy number variants (CNVs) are robustly associated with autism spectrum disorder (ASD) and other developmental neuropsychiatric disorders, such genetic variants provide a genetically homogeneous and identifiable

These authors contributed equally: Mitsuteru Nakamura, Kenny Ye, Mariel Barbachan e Silva

Supplementary information The online version contains supplementary material available at https://doi.org/10.1038/s41380021-01089-y.

Noboru Hiroi

hiroi@uthscsa.edu

Extended author information available on the last page of the article entry point toward a better understanding of psychiatric disorders [1-3]. However, highly variable developmental trajectories remain a major challenge. Not all CNV carriers are diagnosed with developmental psychiatric disorders (i.e., incomplete penetrance), and the precise disorder and their symptomatic severity vary considerably (i.e., variable expressivity).

Mouse models of CNVs-despite caveats concerning differences among species-allow the impact of gene dosage on behavioral dimensions to be experimentally manipulated and isolated from extraneous variables. Moreover, mouse models provide a technical means to prospectively identify, within 1 month, neonatal signs that predict later behavioral abnormalities, which require a much longer period for humans. Many $\mathrm{CNV}$ mouse models with well-controlled genetic backgrounds (e.g., 1q21.1, 3q29, 15q11.13, maternal 15q11-13, 16p11.2, and 22q11.2) often do not exhibit 
deficits in social behaviors and other behavioral dimensions relevant to developmental neuropsychiatric disorders, however [4-12].

Among early predictors, high-pitched cries during infancy are the earliest incipient sign of later diagnosis of idiopathic cases of ASD in humans [13-15]. In mice, atypical neonatal vocalizations of pups with an ASD risk gene variant do not elicit optimal maternal behaviors [16] and are considered an integral deficit of social communication $[13,17]$. However, due to weak or absent deficits in social behaviors in mouse models of $\mathrm{CNV}$, it has been difficult to determine the predictive value of neonatal vocalization for later social behaviors.

Carriers of hemizygous deletion at human chromosome 16 p11.2 exhibit many developmental neuropsychiatric disorders [18, 19]; approximately one-fifths of 16p11.2 deletion carriers are diagnosed with ASD [19]. Children with 16p11.2 deletion variably show atypical developmental trajectories of motor, social, and cognitive dimensions [20]. Arbogast et al. [21] generated the first coisogenic mouse model of $16 \mathrm{p} 11.2$ deletion, in which $\mathrm{ES}$ cells derived from C57BL/6N mice were used for gene targeting and the same inbred mouse line was used as a breeder. This study elegantly addressed the interpretative limitation of earlier pioneering work that used noncongenic models. Like other coisogenic and congenic CNV mouse models $[6,12]$, this coisogenic model exhibited no abnormality in social interaction or preference. The genuine contribution of this and other CNVs to phenotypes relevant to ASD and link between neonatal signs and later phenotypes remains unclear.

As many extraneous variables in testing environments mask or exaggerate phenotypes in mice [22, 23], we developed an independent line of coisogenic mouse model of proximal 16p11.2 hemizygous deletion. Our deepphenotyping and computational approaches identified call types and call sequences of neonatal social communication that predict postpubertal social behaviors in a genotypedependent manner even in this largely asymptomatic model. Our approaches provide a technical means to determine the developmental trajectories with variables within dimensions of developmental psychiatric disorders.

\section{Methods and materials}

The actual experiments were conducted while Noboru Hiroi was at Albert Einstein College of Medicine. Animal handling and use followed the protocols that were approved by the Animal Care and Use Committees of Albert Einstein College of Medicine, in accordance with NIH guidelines. The analysis and manuscript were completed at the University of Texas Health Science Center at San Antonio where Noboru Hiroi is currently employed.
We developed a coisogenic mouse model of $16 \mathrm{p} 11.2$ deletion (see Supplementary Information (SI), Mouse and RNA-seq). We recorded and analyzed their behaviors during the neonatal period (P8 and P12) [24], and during the postpubertal period starting at the age of 1 month (see SI, Behavioral Analyses). The minimal sample size was determined by power analyses based on our previous study [16]. Data were computationally analyzed using the Lasso regression model, random forest, Markov model, and linear regression model (see SI, Computational Analyses).

We compared group means using analysis of variance, followed by Newman-Keuls post hoc tests, if interaction was significant. Two-sided $t$-tests were used when there were only two groups. A probability of $\leq 0.05$ was considered significant. When multiple tests were applied to a dataset, the significance level was adjusted using Benjamini-Hochberg's correction. When either the assumption homogeneity of variance or normality was violated, data with a repeated measure were analyzed by a generalized linear mixed model; for comparisons of a pair of data, nonparametric tests were used.

\section{Results}

\section{Coisogenic mouse model of 16p11.2 deletion}

We developed a coisogenic mouse model of human proximal 16 p11.2 hemizygous deletion through in vitro Cre-mediated recombination of a $378 \mathrm{~kb}$ region of the $7 \mathrm{qF} 3$ region spanning from Mapk3 to Spn genes (Fig. S1A). RNA-seq analysis confirmed that the expression of genes encoded in the deleted region was reduced in the targeted region (Fig. S1B). However, reduction of some genes did not reach statistical significance in some brain regions due to large variance (e.g., Tbx6, Pagrla, Pagrlb, and Zgl6) or very low baseline expression (i.e., floor effect).

\section{Characterization of neonatal vocalizations and postpubertal social behaviors}

As each genetic variant is likely to cause unique-as well as common-phenotypic features, finding a phenotype is fundamentally exploratory and does not permit a priori hypotheses. Because of this, mice were tested for neonatal vocalizations and then for a wide range of other behavioral dimensions thought to be relevant to ASD and other developmental neuropsychiatric disorders, at 1 month of age, when mice start to show early signs of puberty [25].

Neonatal Del/ + and $+/+$ littermates were indistinguishable at postnatal $(\mathrm{P})$ day 8 and $\mathrm{P} 12$ in the average numbers of each call type (Fig. 1A) and in all call types (Fig. 1A, inset). At the age of 1 month, Del/ + mice and $+/+$ littermates were 

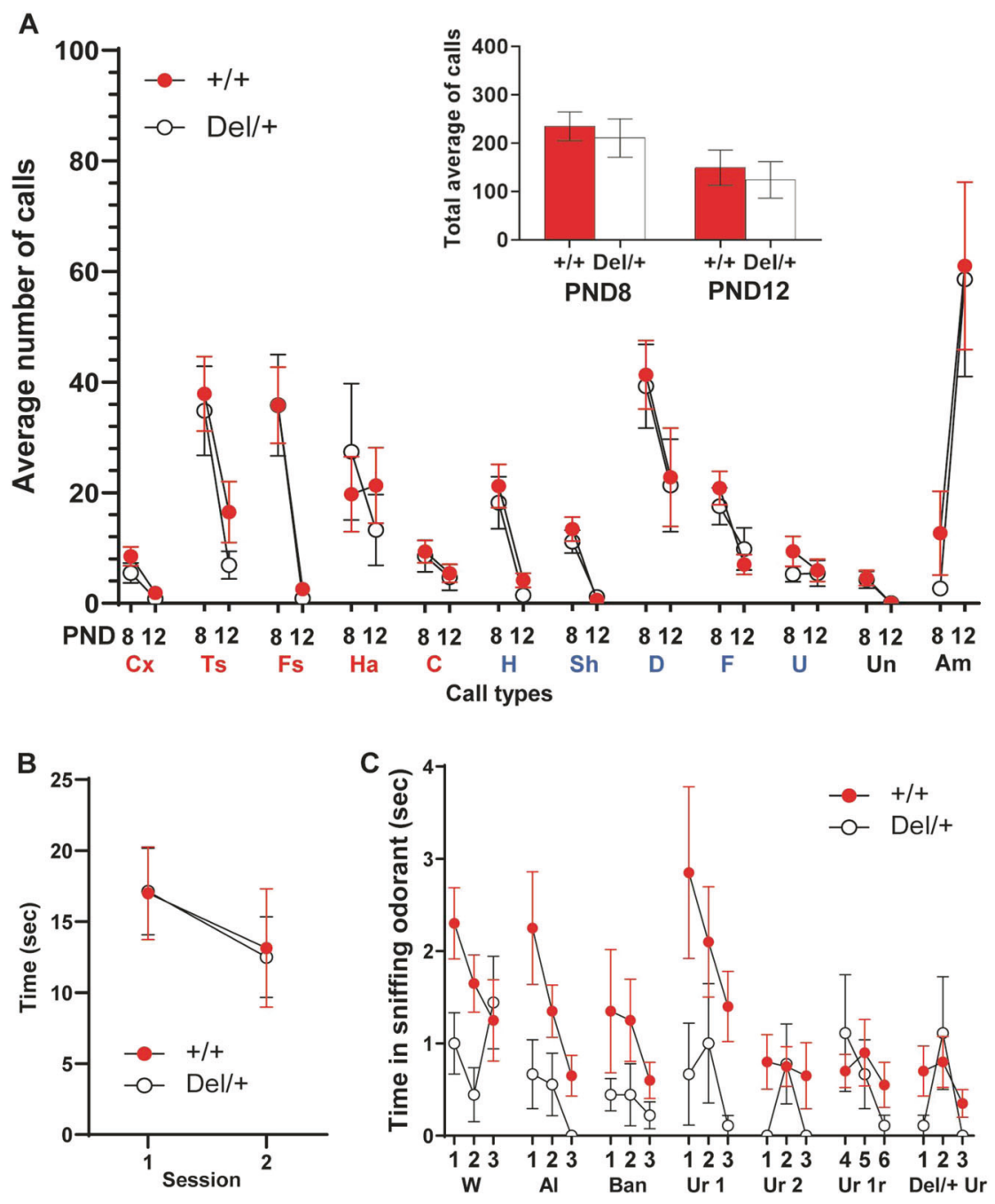

Fig. 1 Neonatal and postpubertal social behaviors. A The average $(+\mathrm{SEM})$ is shown. $+/+$ and Del/ + mice were indistinguishable in the total number of each call type emitted during the 5-min recording period at postnatal days 8 and 12. A mixed model analysis showed no effect of genotype or its interaction with other factors (genotype, $F(1,56.285)=$ $0.085, p=0.771$; genotype $\times$ age, $F(1,1199.982)=0.204, p=0.652$; genotype $\times$ call type, $F(11,1137.054)=0.150, p=0.999$; genotype $\times$ age $\times$ call type, $F(11,1137.054)=0.303, p=0.985)$. Inset: $+/+$ and $\mathrm{Del} /+$ mice were indistinguishable in total number of calls emitted during the 5-min recording period (Mann-Whitney test, P8, $U=386$, $p=0.5535 ; \mathrm{P} 12, U=207, p=0.8026) .+/+: \mathrm{P} 8, N=37 ; \mathrm{P} 12, N=29$. $\mathrm{Del} /+, \mathrm{P} 8, N=23 ; \mathrm{P} 12, N=15$. Multiple-wave call types (red): $\mathrm{Cx}$ complex, Ts two syllable, Fs frequency steps, Ha harmonics, C composite. Simple call waves (blue): H hump, Sh short, D downward, F flat, U upward. Other types (black): A ambiguous, Un uncharacterized. B Time (means \pm SEM) spent in active, affiliative social interaction is shown in two successive 5-min sessions at 1 month of age

(Mann-Whitney test; Session 1, $U=50, p=0.7008$; Session 2, $U=$ 44.5, $p=0.4495) .+/+, N=14 ; \mathrm{Del} /+, N=8 . \mathbf{C}+/+$ mice spent more time (mean $(\mathrm{s}) \pm \mathrm{SEM})$ in sniffing at an Eppendorf tube containing each odorant at Trial 1 and exhibited a higher degree of habituation to each odorant than Del/ + mice at 1 month of age. As the assumption of normality in all cases except for Del/ + , water, Trial 1, and of homogeneity of variance in 11 out of 21 comparisons between $+/+$ and Del/ + was violated, we used a generalized linear mixed model. $+/+$ and Del/ + mice differ (genotype, $F(1,27)=8.966, p=0.006$; odorant, $F(6,540)=$ $3.795, p=0.001$; trial, $F(2,540)=5.747, p=0.003$; genotype $\times$ odorant, $F(6,540)=1.727, p=0.113$; genotype $\times$ trial, $F(2,40)=1.221$, $p=0.296$; genotype $\times$ odorant $\times$ trial, $F(12,540)=0.583, p=0.857)$. $\mathrm{W}$ water, $\mathrm{Al}$ almond odorant, Ban banana odorant, Ur 1 urine of one male C57BL/6J mouse, Ur 2 urine of another male C57BL/6J mouse, Ur1r a second exposure to the urine of the first male C57BL/6J mouse (urine 1), DEL/+Ur urine of one male nonlittermate Del/+mouse. $+/+$, $N=20 ; \mathrm{Del} /+, N=9$.

indistinguishable in reciprocal social behavior (Fig. 1B). However, Del/+ mice exhibited fewer olfactory responses to both nonsocial and social odorants than $+/+$ mice (Fig. 1C).

By contrast, Del/ + mice were indistinguishable from $+/+$ littermates in other standard behavioral assays at 1 month of age: approach to a novel, nonsocial object and its habituation
(Fig. S2A), anxiety-related behavior in an elevated plus maze (Fig. S2BC), acoustic startle (Fig. S2D), prepulse inhibition (Fig. S2E), working memory/repetitive behavioral traits in a T-maze (Fig. S2F), motor behavior (Fig. S2G), and thigmotaxis (Fig. S2H). Mice were additionally tested at 2 months for working memory only, as severe working memory deficits 
often appear later during development in mouse models of genetic variants associated with ASD [26, 27] and humans with ASD [28-34]. Del/t and $+/+$ mice were indistinguishable in this task at 2 months of age (data not shown). The phenotypes in olfactory responses (see Fig. 1C) are not due to altered responses to an object or anxiety responses to novel objects, as Del/ + and $+/+$ mice were indistinguishable in their responses to a novel object (see Fig. S2A) or anxietyrelated traits (see Fig. S2B, C, H). Consistent with the previous report of another coisogenic mouse model of $16 \mathrm{p} 11.2$ [21], our Del/+ model was significantly underweight compared to $+/+$ mice throughout development (Fig. S3). However, this physical developmental delay did not impact any neonatal or postpubertal behaviors, except for postpubertal olfactory responses (see Figs. 1A, B and S2A-H).

\section{Computational extraction of predictive variables of neonatal vocalizations for postpubertal social behaviors}

We ran least absolute shrinkage and selection operator (Lasso) regression model on the data to extract predictive features (Fig. S4, predictive model, Lasso regression model 1 ), as it is ideal to extract a small number of most robust predictive features from a large pool of collinear parameters when the number of variables is greater than the number of observations. The candidate explanatory variables were genotype, acoustic parameters, number and ratios of distinct call types, and number and probabilities of distinct call transitions (i.e., sequences). The communicative capacity of neonatal vocalization sequences and their relevance to ASD have been demonstrated in a mouse model of a genetic variant linked to developmental neuropsychiatric disorders $[16,35]$. The dependent variables were social interaction at Session 1, its habituation from the first to last session (i.e., Session 1-Session 2), olfactory responses to the urine smell at Trial 1, and its habituation from the first to last trial (i.e., Trial 1-Trial 3).

Each postpubertal social behavior had its unique predictive signature of neonatal calls (Fig. 2; Table S1). Many selected predictors were the transition probabilities and numbers of transitions from one call to another (e.g., Fs $\rightarrow$ D (\#) and $\mathrm{A} \rightarrow \mathrm{F}$ for social interaction; $\mathrm{H} \rightarrow \mathrm{Ha}$ for social habituation; $\mathrm{U} \rightarrow \mathrm{C}$ for olfactory response and olfactory habituation) (Table S2). The ratios of some call types also were selected (e.g., U(R) for social interaction; $\mathrm{Ha}(\mathrm{R})$ for social habituation; $\mathrm{C}(\mathrm{R})$ for olfactory response). The identified call features had good predictive values for the four readouts of behaviors of $+/+$ mice, but not Del $/+$ mice (see Table S2).

One possibility for this observation is that neonatal call features predictive of postpubertal social behaviors are less robust in Del/ + than in $+/+$ mice. To find neonatal call features that are predictive of postpubertal social behaviors in Del/+ mice, we carried out another Lasso regression with genotype as the dependent variable (Fig. S4, Lasso regression model 2). This model selected a unique set of call features as predictors (Fig. S5). Random forest, with Del/+ as a positive reference dependent variable, showed high specificity (0.7917) (Table S3), indicating that the genotype $+/+$ can be fairly accurately identified by certain call features (i.e., a low false positive rate). In contrast, the predictors had low sensitivity (0.5000) and sensitivity and specificity are significantly different (McNemar's test, $p=1.012 \times 10^{-5}$ ); the selected features often misjudged Del/ + mice as $+/+$ (i.e., false negative). As a result, the overall accuracy was significant $\left(p=8.837 \times 10^{-8}\right)$ but modest $(0.681)$, suggesting that there are proportionally more neonatal call features predictive of $+/+$ genotype than Del/ + genotype. Some of the selected features predicted social behaviors in a genotype-dependent manner (Table S4).

The probabilities and numbers of call sequences were extracted as predictors for postpubertal behaviors more often than any other variables (see Fig. 2) and for genotype (see Fig. S5). To further explore call sequences, we used Shannon entropy analysis, sparse partial least squares discriminant analysis (sPLS-DA), and Markov models (see Fig. S4). Shannon entropy analysis determined the degree of randomness in how many call types pups used (H0), how often pups nonrandomly chose call types within their repertoire $(\mathrm{H} 1)$, or how pups nonrandomly chose call types in two-call $(\mathrm{H} 2)$, three-call $(\mathrm{H} 3)$, and four-call sequences (H4) (Fig. 3A). $+/+$ and Del/ + differed at $\mathrm{H} 2$ and $\mathrm{H} 3$ of P8, but not at any level of P12.

We next applied sPLS-DA analysis to select variables based on their relative contribution to classification of genotype to examine individual and group-level variability using two-call sequences. Individual mice are plotted in the two-component model space (Fig. 3B). Component 1 separated pups in terms of age, which represented a developmental change in call sequences. In Component 2, the two genotype groups did not well segregate, but more individually separated at P8 than P12, indicating larger individual variability in the use of specific two-call sequences at P8 than at $\mathrm{p} 12$.

While the sPLS-DA analysis revealed individual variability in two-call transitions, there might be some call sequences that are frequently emitted by either genotype. We thus applied Markov models to two-call sequences within each genotype. Call sequences frequently emitted by $+/+$ pups were $\mathrm{Ts} \rightarrow \mathrm{Ts}, \mathrm{Fs} \rightarrow \mathrm{Fs}, \mathrm{U} \rightarrow \mathrm{F}$, and $\mathrm{A} \rightarrow \mathrm{A}$, while call sequences frequently emitted by $\mathrm{Del} /+$ pups were $\mathrm{Ha} \rightarrow \mathrm{Ha}$, $\mathrm{Ts} \rightarrow \mathrm{F}, \mathrm{H} \rightarrow \mathrm{D}$, and $\mathrm{C} \rightarrow \mathrm{F}$; other call sequences $(\mathrm{F} \rightarrow \mathrm{F}$, $\mathrm{Ha} \rightarrow \mathrm{H}$, and $\mathrm{Fs} \rightarrow \mathrm{F}$ ) were frequently emitted in both $+/+$ and Del/ + pups (Fig. 3C). $\mathrm{Ha} \rightarrow \mathrm{H}$ and $\mathrm{H} \rightarrow \mathrm{D}$ had significant 


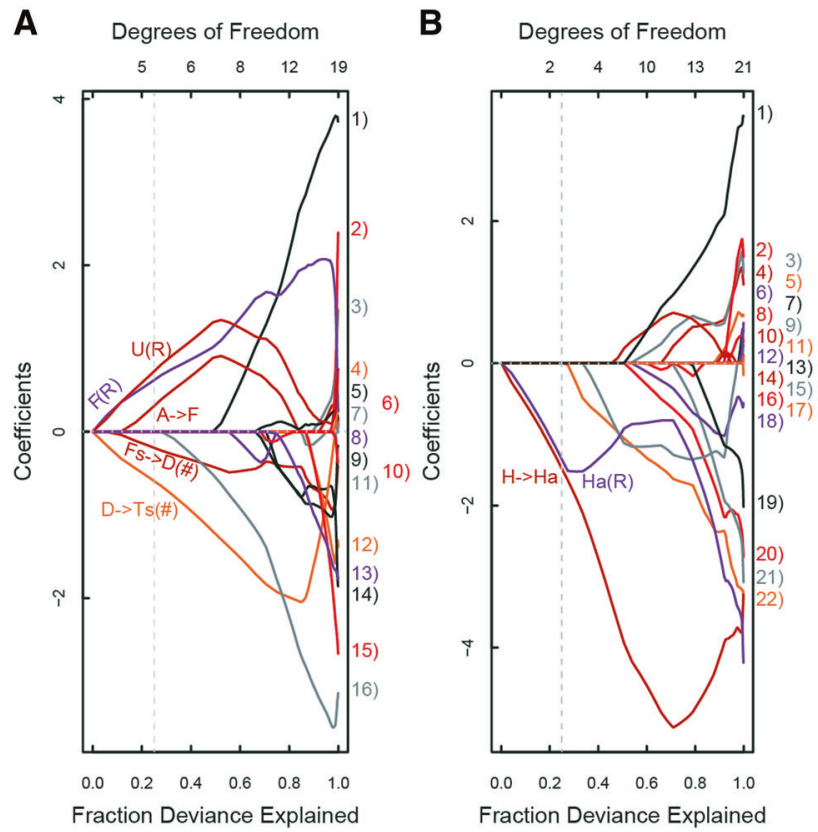

Fig. 2 Fraction deviance explained and coefficients of variables determined by Lasso regression model 1 (see Fig. S4). All parameters (call type number and ratios, call sequence number and probabilities, acoustic parameters) were used for selection. A Social interaction. B Social habituation (Session 1 score - Session 2 score). C Olfactory response. D Olfactory habituation (Trial 1 score - Trial 3 score). Cutoff was set at 0.25 of fraction deviance explained (see light gray vertical broken line). The probabilities or number (\#) of two-call sequences and ratio $(\mathrm{R})$ of the number of a call type emitted to all calls emitted were selected (see Table S1 for detailed labels). Lasso extracts features along

model fit as predictors for social interaction and olfactory habituation, respectively, in Del/+ (Table S5).

Our comprehensive feature extraction (see Fig. S4, Lasso regression models 1 and 2) did not detect acoustic parameters as robust predictors except for maximum frequency minimum parameter of the entire call (MaFmi) for genotype (see Fig. S5). Random forest (see Fig. S4, Radom Forest 2) showed that quantitative acoustic features yielded a low level of accuracy (0.5642) with low sensitivity (0.4946) and modest specificity (0.6338) (Table S6) for genotype, confirming that acoustic parameters are not good predictors.

Among all call features extracted from Lasso and linear regression models, there were neonatal call sequences and call types that are positively or negatively correlated with postpubertal social behaviors (Fig. S6). For example, high and low probabilities of neonatal $\mathrm{A} \rightarrow \mathrm{F}$ and Fs-D(\#), respectively, predict high levels of postpubertal social interaction in $+/+$ mice; the higher $\mathrm{Ha} \rightarrow \mathrm{H}$ is, the higher their postpubertal social interaction is in $\mathrm{Del} /+$ mice (Fig. S6A). $\mathrm{H} \rightarrow \mathrm{Ha}, \mathrm{Ha}(\mathrm{R})$, and $\mathrm{Ha} \rightarrow \mathrm{Ha}$ negatively predicted social habituation in $+/+$ mice (Fig. S6B). $U \rightarrow C$, $\mathrm{F} \rightarrow \mathrm{D}$, and $\mathrm{C}(\mathrm{R})$ in $+/+$ and $\mathrm{D} \rightarrow \mathrm{U}(\#)$ in $\mathrm{Del} /+$ positively predicted olfactory response (Fig. S6C). $\mathrm{U} \rightarrow \mathrm{C}$ and $\mathrm{Sh} \rightarrow \mathrm{H}$ in
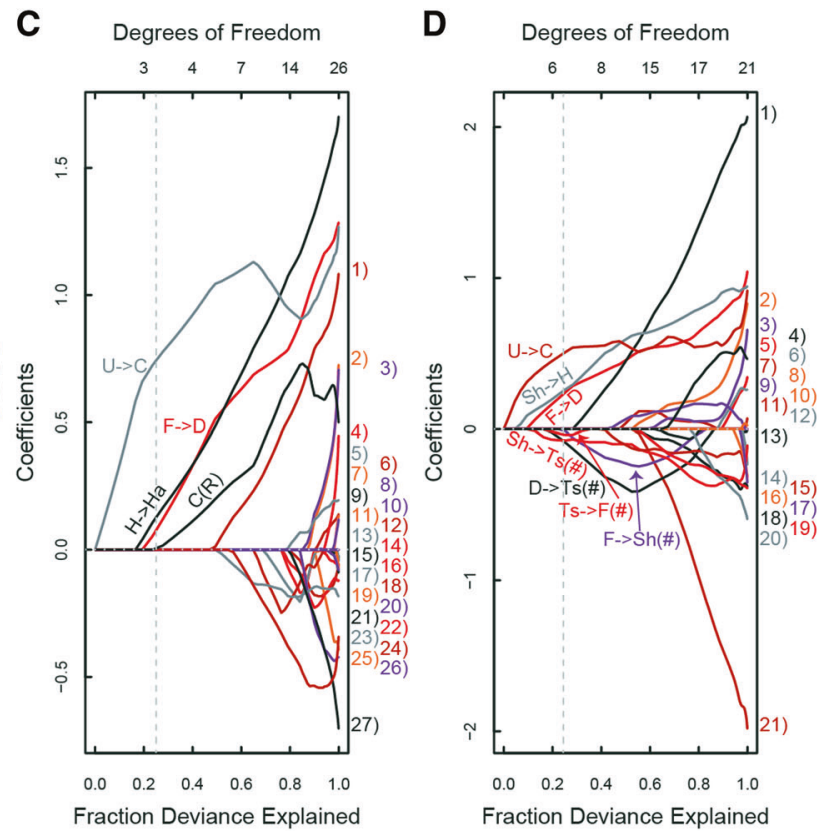

the proportion of deviance explained. The regularization parameter lambda decreases as the proportion of deviance explained increases. Thus, large lambda values (i.e., small values along the axis of the proportion of deviance explained) are robust predictors. $\mathrm{Cx}$ complex, Ts two syllable, Fs frequency steps, Ha harmonics, C composite. H hump, Sh short, D downward, F flat, U upward. Other types: A ambiguous, Un uncharacterized. The number of mice that completed social interaction or olfactory response testing and emitted more than ten calls at P8 were $+/+, N=11 ; \mathrm{Del} /+, N=8$ for social interaction and $+/+, N=16$; $\mathrm{Del} /+, N=8$ for olfactory response.

$+/+$ mice and $\mathrm{D} \rightarrow \mathrm{U}(\#)$ and $\mathrm{H} \rightarrow \mathrm{D}$ in $\mathrm{Del} /+$ mice positively predicted olfactory habituation; $\mathrm{D} \rightarrow \mathrm{Ts}(\#), \mathrm{Sh} \rightarrow \mathrm{Ts}(\#)$, and $\mathrm{Ts} \rightarrow \mathrm{F}(\#)$ negatively predicted olfactory habituation in $+/+$ mice (Fig. S6D).

Our analyses collectively identified many paths from specific calls and call sequences to postpubertal social behaviors in a genotype-dependent manner (Fig. 4). Several patterns emerged. First, there is an overall shift of predictors from call types with multiple waves (Fig. 4, red call type bars) to simple call types (Fig. 4, blue call type bars) in Del/+ mice. Second, nonidentical sets of neonatal call features were predictors for the four postpubertal social behavioral readouts (see Figs. 4 and S6). Third, all predictors were genotype-specific within each postpubertal social behavioral readout (see Fig. 4 and Tables S2, S4, and S5). Fourth, some call features predicted more than one postpubertal social behaviors (see Fig. 4 and $\mathrm{U} \rightarrow \mathrm{C}$ in Fig. S6A, C, D; D $\rightarrow \mathrm{Ts}(\#)$, Fig. S6A, D; D $\rightarrow \mathrm{U}(\#)$, Fig. S6C, D).

That call sequences are most frequently identified as predictors among all neonatal call variables further strengthens our hypothesis that ASD risk gene variants have a negative functional impact on social communication between pups and mothers through altered call sequences $[16,35]$. 
A

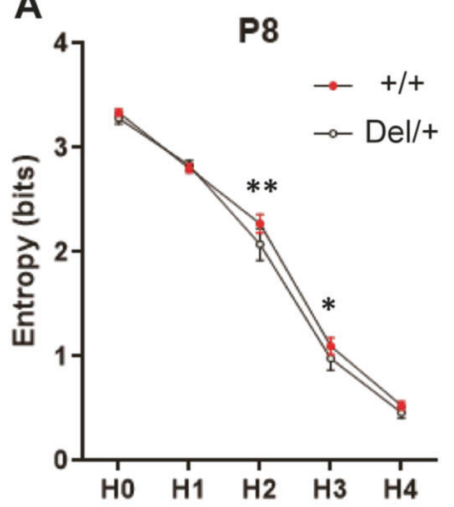

C

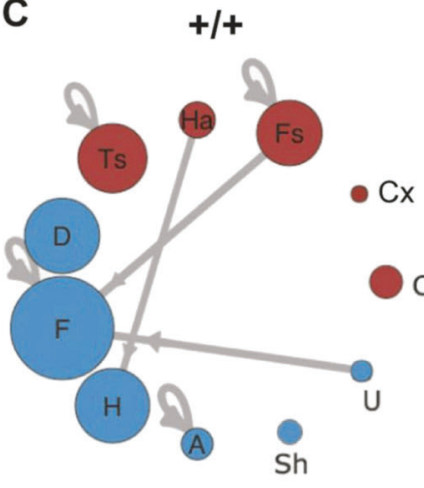

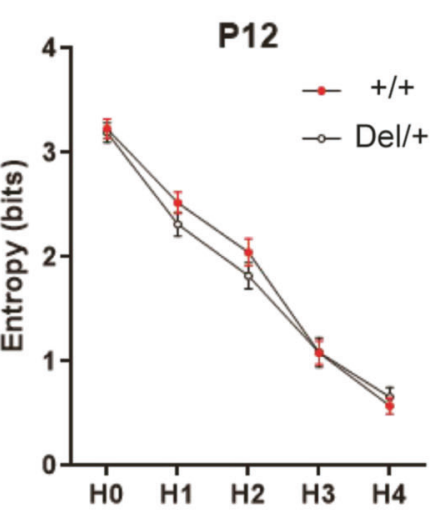

Del/+

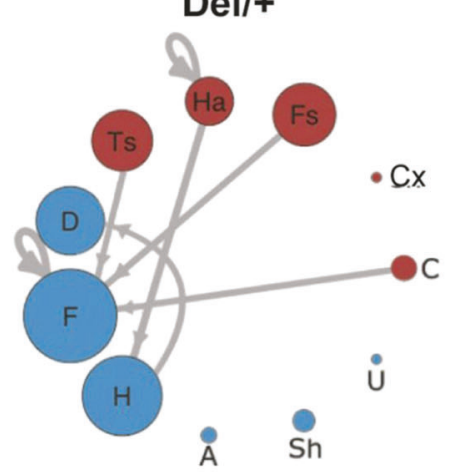

B

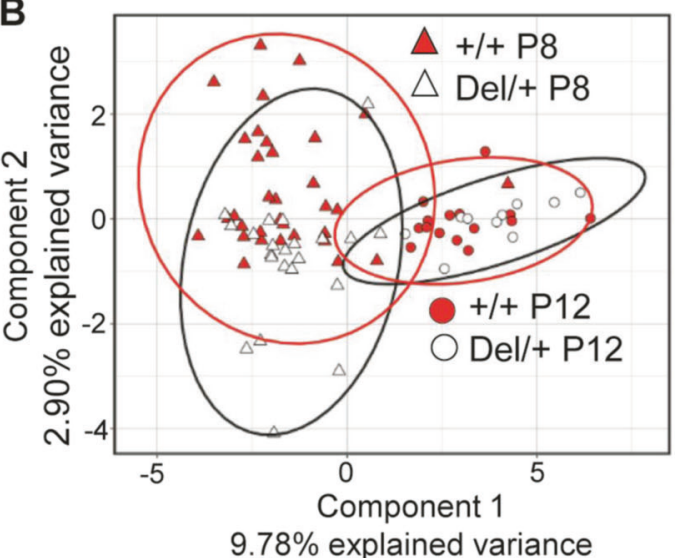

P8

Fig. 3 Sequence structures of neonatal calls. A Shannon entropy analysis of the randomness of call repertoires (H0), of individual calls used within the repertoire (H1), of two-call sequence $(\mathrm{H} 2)$, three-call sequence $(\mathrm{H} 3)$, and four-call sequence $(\mathrm{H} 4) .+/+$ mice and Del/ + mice differed at $\mathrm{H} 2(p=0.00761)$ and $\mathrm{H} 3(p=0.04467)$ at P8, as determined by linear mixed model fitted using REML with $t$-test degrees of freedom approximated using Satterthwaite's method. * $p<0.05$; ** $p<$ 0.001. B sPLS-DA analysis of call. Two factors segregated two-call

\section{Discussion}

Deep-phenotyping analyses showed that our coisogenic mouse model of 16p11.2 deletion generally lacks behavioral phenotypes in standard dimensional measures that are considered relevant to ASD. However, two subdimensional features differentiated genotypes: (1) frequently emitted neonatal call sequences and (2) olfactory responses to nonsocial and social odor. Our computational analyses identified neonatal call types and sequences that differentially predicted individual levels of postpubertal social interaction and olfactory responses to a social cue in a genotype-dependent manner. These data reveal a genotypespecific hidden structure in developmental trajectories from neonatal social communication to postpubertal social behaviors. As many well-controlled mouse models of genetic risk factors show weak or few phenotypic abnormalities in standard measures [6], our approach provides a novel means to identify hidden subdimensions and sequences in terms of age (Component 1) and, to a lesser extent, genotype of P8 data (Component 2). C Markov models identified the most frequent call connections between two calls at P8. Multiple-wave call types (red): Cx complex, Ts two syllable, Fs frequency steps, Ha harmonics, $\mathrm{C}$ composite. Simple call waves (blue): H hump, Sh short, D downward, $\mathrm{F}$ flat, $\mathrm{U}$ upward, A ambiguous, Un uncharacterized. + I+: P8, $N=33 ; \mathrm{P} 12, N=22$. Del/+: P8, $N=16 ; \mathrm{P} 12, N=10$.

altered developmental paths in apparently asymptomatic mouse models of not just 16p11.2 CNV but also of many other genetic risk variants for developmental neuropsychiatric disorders.

Our model isolated effects of $16 \mathrm{p} 11.2$ deletion against a homogeneous genetic background; therefore, any phenotypic difference can be ascribed to the deletion. Consistent with observations in another coisogenic mouse model of $16 \mathrm{p} 11.2$ deletion with a C57BL/6N background tested between 3 and 4 months of age [21], our 16p11.2 deletion model was indistinguishable from $+/+$ littermates in the total number of calls or of each call type during the neonatal period and prepulse inhibition, reciprocal social interaction, working memory, and repetitive behavioral traits in spontaneous alternation, anxiety-related behaviors in the elevated plus maze, and thigmotaxis and locomotor activity in an open field at the age of 1 month; their coisogenic model with C57BL/6N background was not tested for olfactory responses. While the lack of working memory deficits was 


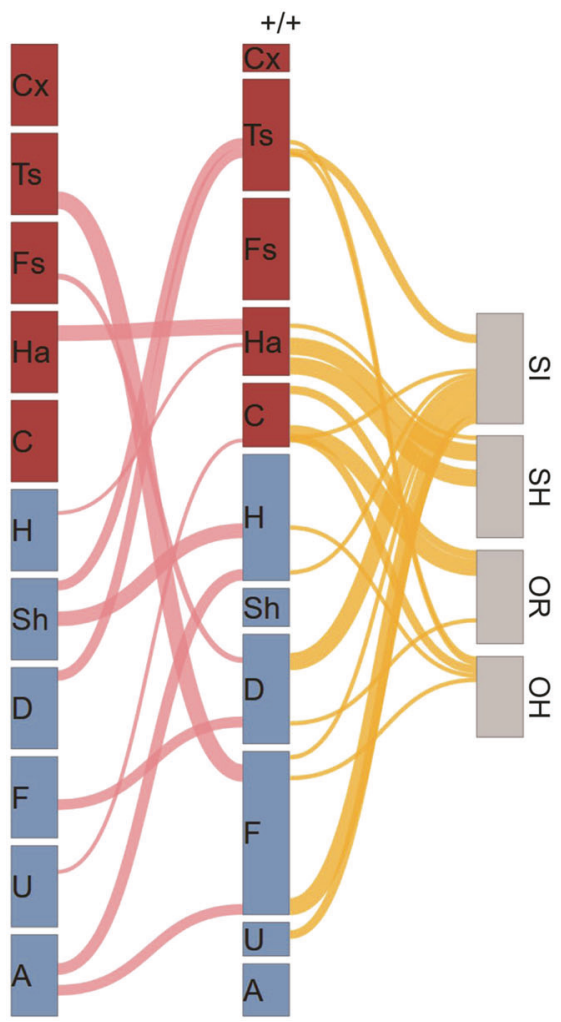

Fig. 4 Many paths from neonatal call types and sequences to postpubertal social behaviors. The thickness of each path from one call type to the next represents the proportion of such transition probabilities from one call to another (see pink lines). Only significant correlation coefficients $(p<0.05)$ are shown (see Tables S2, S4, and S5). The strength of paths predicting each postpubertal social behavior is shown as thickness of lines from the second call column to

consistent with what is seen in individuals with $16 \mathrm{p} 11.2$ deletions [36], apparently normal reciprocal social interaction in our and their mouse models is inconsistent with high rates of ASD in 16p11.2 deletion carriers. Although individuals with 16p11.2 deletions have not been characterized for neonatal cries or olfactory response, idiopathic cases of ASD exhibit abnormalities in neonatal cries [13] and olfactory responses [37, 38], but are normal in prepulse inhibition under the standard test condition [39-43].

The general lack of phenotypes in our and their coisogenic mouse models of $16 \mathrm{p} 11.2$ deletion could be interpreted as suggesting that developmental neuropsychiatric disorders seen at elevated rates among 16p11.2 deletion carriers are not primarily caused by this chromosomal deletion alone. This $\mathrm{CNV}$ might require other coexisting genetic variants, including second CNVs [44] and common genetic variation [21, 45], and environmental insults, such as preterm and C-section birth [46] to be fully symptomatic. Alternatively, this $\mathrm{CNV}$ might manifest its impacts on subdimensions that are predominantly used for certain functions in a given species. Our observation underscores

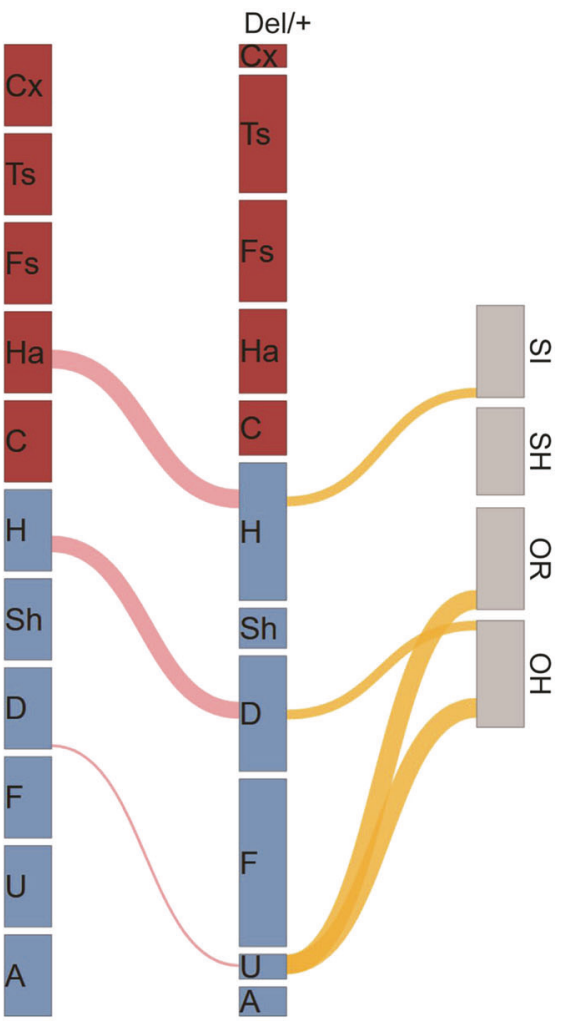

postpubertal social behavior. Only cases in which goodness of fit was significant (Tables S2, S4, and S5) are shown (see yellow lines). The thickness of lines with different levels of significance $(p<0.05, p<$ 0.01 , and $p<0.001)$ are represented by an arbitrary thickness unit $(x)$, $2 x$, and $4 x$, respectively. Cx complex, Ts two syllable, Fs frequency steps, Ha harmonics, C composite, H hump, Sh short, D downward, F flat, U upward. Other types: A ambiguous, Un uncharacterized.

the importance of exploratory, deep behavioral phenotyping to identify the phenotypic points within a dimension at which the impacts of genetic variants appear.

Our coisogenic mouse model of 16p11.2 was insensitive to the presentation of new nonsocial and social odorants. This phenotype does not reflect nonspecific sensory or motivational deficits to respond to a social cue, a novel object or anxiety-evoking stimuli (see Fig. 1B; Fig. S2A-C, $\mathrm{H})$. As Del/+ mice are also impaired in their responses to nonsocial odorants (e.g., water), it is still possible, however, that $16 \mathrm{p} 11.2$ hemizygosity impairs olfactory sensation, motivation to respond to nonsocial and social olfactory cues, or both. Altered responses to various nonsocial and social olfactory stimuli have been noted in mouse models of other genetic variants associated with ASD [47, 48]. In humans, individuals with idiopathic ASD respond abnormally to both nonsocial and social olfactory stimuli [37, 38, 49]. Individuals with ASD do not differentiate between social and nonsocial odorants [38] and have generally blunted differentiating responses to pleasant and aversive nonsocial odor, and this lack of differentiating 
response to nonsocial odorants is correlated with their social deficits [37]. Thus, while the exact nature of altered olfactory responses in genetic mouse models of ASD, including ours, remains unclear, defective olfactory responses to both nonsocial and social odorants are a dimension of ASD.

We do not rule out the possibilities that $16 \mathrm{p} 11.2$ hemizygosity indirectly impacted neonatal call sequences and olfactory responses via craniofacial abnormalities and a developmental delay in body weights, respectively. However, it is difficult to explain why craniofacial abnormalities did not alter the frequencies and duration of various call types in Del/+ pups and why the developmental delay had no impact on any other social and cognitive behaviors (i.e., social interaction, novel object approach, and working memory). More work is needed to explore the possibilities that altered sequences of neonatal vocalizations and altered olfactory responses are manifestations of a shared physical or neuronal abnormality at different developmental stages.

Our data are inconsistent with reported data of noncongenic models of $16 \mathrm{p} 11.2$ deletion. Those models were reported to not differ from their wild-type littermates in olfactory responses to social or nonsocial cues [50-52]. Furthermore, noncongenic models of 16p11.2 deletion were reported to emit more downward and short calls and fewer frequency steps in neonatal vocalizations than their wildtype littermates [51]. Several likely sources of this apparent inconsistency include sex, age of testing, and housing conditions. Another fundamental difference is the degree of homogeneity of genetic background. When mutant mice are not backcrossed to an inbred strain for ten or more generations, noncongenic mutant mice carry, at the chromosomal loci flanking the deleted segment, more alleles of mouse strain of ES cells (e.g., 129SvJ) than those derived from a breeder strain; in contrast, wild-type littermates carry more alleles of a breeder strain (e.g., C57BL/6J) at the same loci than mutant mice. These different genetic backgrounds systematically persist among many cohorts due to a low rate of recombination between the deleted locus and nearby loci. Such a confounding factor results in behavioral, neuronal, cellular, and molecular phenotypic differences that might not be attributable to the mutated gene [6,53-57]. Use of commercially available B6129SF1/J mice does not serve as a valid control for this confounding factor either, as a mutant mouse is not an F1 generation and still carries more ES cell alleles at the flanking loci than such "control" mice, thereby still creating consistent and systematic differences in the genetic backgrounds between such "wild-type control" and mutant littermates [6]. Moreover, the absence of phenotypic differences in noncongenic models poses an additional interpretative issue, as the baselines of many behavioral and neural phenotypes differ widely among inbred mouse strains [57]. If systematic enrichment of ES cell alleles in mutant mice causes a higher baseline than wild-type mice, any reduction in scores due to the targeted mutation might simply result in an apparently normal phenotype.

Our computational approaches have translational value. Human babies who are later diagnosed with idiopathic ASD (i.e., incipient ASD babies) exhibit atypical cries [13], but the trajectories of dimensions from the neonatal period to later diagnosis of ASD are heterogeneous and unstable [58-61]. Our approach to focus on variables within dimensions, instead of categorical classification of ASD or dimensions per se, could be useful in determining the structure of developmental trajectories in both $\mathrm{CNV}$-associated and idiopathic cases of ASD in humans, as well as in genetic mouse models of CNVs.

\section{Data availability}

All data and programs will be provided upon request.

Acknowledgements We thank Ms. Patricia Pouso for call type analyses. Research reported in this publication was partly supported by the National Institutes of Health (R01MH099660 and R01DC015776) and funds from Astellas Pharma, Inc. to NH. The content is solely the responsibility of the authors and does not necessarily represent the official views of the National Institutes of Health. The open access license has been selected.

\section{Compliance with ethical standards}

Conflict of interest The authors declare no competing interests.

Publisher's note Springer Nature remains neutral with regard to jurisdictional claims in published maps and institutional affiliations.

Open Access This article is licensed under a Creative Commons Attribution 4.0 International License, which permits use, sharing, adaptation, distribution and reproduction in any medium or format, as long as you give appropriate credit to the original author(s) and the source, provide a link to the Creative Commons license, and indicate if changes were made. The images or other third party material in this article are included in the article's Creative Commons license, unless indicated otherwise in a credit line to the material. If material is not included in the article's Creative Commons license and your intended use is not permitted by statutory regulation or exceeds the permitted use, you will need to obtain permission directly from the copyright holder. To view a copy of this license, visit http://creativecommons. org/licenses/by/4.0/.

\section{References}

1. Matsumoto M, Walton NM, Yamada H, Kondo Y, Marek GJ, Tajinda K. The impact of genetics on future drug discovery in schizophrenia. Expert Opin Drug Discov. 2017;12:673-86.

2. Hiroi N, Yamauchi T. Modeling and predicting developmental trajectories of neuropsychiatric dimensions associated with copy number variations. Int J Neuropsychopharmacol. 2019;22: $488-500$.

3. Hiroi N, Nishi A. Dimensional deconstruction and reconstruction of CNV-associated neuropsychiatric disorders. In: Pletnikov MV, 
Waddington JL, editors. Modeling the psychopathological dimensions of schizophrenia: from molecules to behavior. Vol. 23. London, UK: Elsevier; 2015. p. 285-302.

4. Nilsson SR, Fejgin K, Gastambide F, Vogt MA, Kent BA, Nielsen $\mathrm{V}$, et al. Assessing the cognitive translational potential of a mouse model of the 22q11.2 microdeletion syndrome. Cereb Cortex. 2016;26:3991-4003.

5. Nilsson SR, Celada P, Fejgin K, Thelin J, Nielsen J, Santana N, et al. A mouse model of the $15 \mathrm{q} 13.3$ microdeletion syndrome shows prefrontal neurophysiological dysfunctions and attentional impairment. Psychopharmacology. 2016;233:2151-63.

6. Hiroi N. Critical reappraisal of mechanistic links of copy number variants to dimensional constructs of neuropsychiatric disorders in mouse models. Psychiatry Clin Neurosci. 2018;72:301-21.

7. Saito R, Koebis M, Nagai T, Shimizu K, Liao J, Wulaer B, et al. Comprehensive analysis of a novel mouse model of the $22 \mathrm{q} 11.2$ deletion syndrome: a model with the most common 3.0-Mb deletion at the human 22q11.2 locus. Transl Psychiatry. 2020; $10: 35$.

8. Rutkowski TP, Purcell RH, Pollak RM, Grewenow SM, Gafford GM, Malone T, et al. Behavioral changes and growth deficits in a CRISPR engineered mouse model of the schizophrenia-associated 3q29 deletion. Mol Psychiatry. 2021;26:772-83.

9. Baba M, Yokoyama K, Seiriki K, Naka Y, Matsumura K, Kondo $\mathrm{M}$, et al. Psychiatric-disorder-related behavioral phenotypes and cortical hyperactivity in a mouse model of $3 \mathrm{q} 29$ deletion syndrome. Neuropsychopharmacology. 2019;44:2125-35.

10. Nakatani J, Tamada K, Hatanaka F, Ise S, Ohta H, Inoue K, et al. Abnormal behavior in a chromosome-engineered mouse model for human 15q11-13 duplication seen in autism. Cell. 2009;137: 1235-46.

11. Nielsen J, Fejgin K, Sotty F, Nielsen V, Mork A, Christoffersen CT, et al. A mouse model of the schizophrenia-associated 1q21.1 microdeletion syndrome exhibits altered mesolimbic dopamine transmission. Transl Psychiatry. 2017;7:1261.

12. Forsingdal A, Jorgensen TN, Olsen L, Werge T, Didriksen M, Nielsen J. Can animal models of copy number variants that predispose to schizophrenia elucidate underlying biology? Biol Psychiatry. 2019;85:13-24.

13. Esposito G, Hiroi N, Scattoni ML. Cry, baby, cry: expression of distress as a biomarker and modulator in autism spectrum disorder. Int J Neuropsychopharmacol. 2017;20:498-503.

14. Shultz S, Klin A, Jones W. Neonatal transitions in social behavior and their implications for autism. Trends Cogn Sci. 2018;22: $452-69$.

15. Sheinkopf SJ, Iverson JM, Rinaldi ML, Lester BM. Atypical cry acoustics in 6-month-old infants at risk for autism spectrum disorder. Autism Res. 2012;5:331-9.

16. Takahashi T, Okabe S, Broin PO, Nishi A, Ye K, Beckert MV, et al. Structure and function of neonatal social communication in a genetic mouse model of autism. Mol Psychiatry. 2016;21:1208-14.

17. Kikusui T, Hiroi N. A self-generated environmental factor as a potential contributor to atypical early social communication in autism. Neuropsychopharmacology. 2017;42:378.

18. Hanson E, Bernier R, Porche K, Jackson FI, Goin-Kochel RP, Snyder LG, et al. The cognitive and behavioral phenotype of the $16 \mathrm{p} 11.2$ deletion in a clinically ascertained population. Biol Psychiatry. 2015;77:785-93.

19. Niarchou M, Chawner S, Doherty JL, Maillard AM, Jacquemont $\mathrm{S}$, Chung WK, et al. Psychiatric disorders in children with 16p11.2 deletion and duplication. Transl Psychiatry. 2019;9:8.

20. Bernier R, Hudac CM, Chen Q, Zeng C, Wallace AS, Gerdts J, et al. Developmental trajectories for young children with $16 \mathrm{p} 11.2$ copy number variation. Am J Med Genet B Neuropsychiatr Genet. 2017;174:367-80.
21. Arbogast T, Ouagazzal AM, Chevalier C, Kopanitsa M, Afinowi $\mathrm{N}$, Migliavacca E, et al. Reciprocal effects on neurocognitive and metabolic phenotypes in mouse models of $16 \mathrm{p} 11.2$ deletion and duplication syndromes. PLoS Genet. 2016;12:e1005709.

22. Crabbe JC, Wahlsten D, Dudek BC. Genetics of mouse behavior: interactions with laboratory environment. Science. 1999;284: 1670-2.

23. Yang M, Lewis F, Foley G, Crawley JN. In tribute to Bob Blanchard: divergent behavioral phenotypes of $16 \mathrm{p} 11.2$ deletion mice reared in same-genotype versus mixed-genotype cages. Physiol Behav. 2015;146:16-27.

24. Ó Broin PB, Beckert MV, Takahashi T, Izumi T, Ye K, Kang K, et al. Computational analysis of neonatal mouse ultrasonic vocalization. Curr Protoc Mouse Biol. 2018;8:e46.

25. Bronson FH, Dagg CP, Snell GD. Reproduction. In: Green EL, editor. Biology of the laboratory mouse. 2nd ed. New York: Dover Publications, Inc.; 2007.

26. Suzuki G, Harper KM, Hiramoto T, Funke B, Lee M, Kang G, et al. Over-expression of a human chromosome 22q11.2 segment including TXNRD2, COMT and ARVCF developmentally affects incentive learning and working memory in mice. Hum Mol Genet. 2009; 18:3914-25.

27. Boku S, Izumi T, Abe S, Takahashi T, Nishi A, Nomaru H, et al. Copy number elevation of 22q11.2 genes arrests the developmental maturation of working memory capacity and adult neurogenesis. Mol Psychiatry. 2018;23:985-92.

28. Dumontheil I, Roggeman C, Ziermans T, Peyrard-Janvid M, Matsson H, Kere J, et al. Influence of the COMT genotype on working memory and brain activity changes during development. Biol Psychiatry. 2011;70:222-29.

29. Vorstman JA, Breetvelt EJ, Duijff SN, Eliez S, Schneider M, Jalbrzikowski M, et al. Cognitive decline preceding the onset of psychosis in patients With 22q11.2 deletion syndrome. JAMA Psychiatry. 2015;72:377-85.

30. Kates WR, Olszewski AK, Gnirke MH, Kikinis Z, Nelson J, Antshel KM, et al. White matter microstructural abnormalities of the cingulum bundle in youths with 22q11.2 deletion syndrome: associations with medication, neuropsychological function, and prodromal symptoms of psychosis. Schizophr Res. 2015;161:76-84.

31. Duijff SN, Klaassen PW, de Veye HF, Beemer FA, Sinnema G, Vorstman JA. Cognitive development in children with 22q11.2 deletion syndrome. Br J Psychiatry. 2012;200:462-8.

32. Gur RC, Calkins ME, Satterthwaite TD, Ruparel K, Bilker WB, Moore TM, et al. Neurocognitive growth charting in psychosis spectrum youths. JAMA Psychiatry. 2014;71:366-74.

33. Gothelf D, Eliez S, Thompson T, Hinard C, Penniman L, Feinstein $\mathrm{C}$, et al. COMT genotype predicts longitudinal cognitive decline and psychosis in 22q11.2 deletion syndrome. Nat Neurosci. 2005;8:1500-2.

34. Gothelf D, Schneider M, Green T, Debbane M, Frisch A, Glaser $B$, et al. Risk factors and the evolution of psychosis in $22 \mathrm{q} 11.2$ deletion syndrome: a longitudinal 2-site study. J Am Acad Child Adolesc Psychiatry. 2013;52:1192-203 e1193.

35. Kato R, Machida A, Nomoto K, Kang G, Hiramoto T, Tanigaki K, et al. Maternal approach behaviors toward neonatal calls are impaired by mother's experiences of raising pups with a risk gene variant for autism. Dev Psychobiol. 2020. https://doi.org/10.1101/ 2020.05.21.107540.

36. Hippolyte L, Maillard AM, Rodriguez-Herreros B, Pain A, Martin-Brevet S, Ferrari C, et al. The number of genomic copies at the $16 \mathrm{p} 11.2$ locus modulates language, verbal memory, and inhibition. Biol Psychiatry. 2016;80:129-39.

37. Rozenkrantz L, Zachor D, Heller I, Plotkin A, Weissbrod A, Snitz K, et al. A mechanistic link between olfaction and autism spectrum disorder. Curr Biol. 2015;25:1904-10. 
38. Endevelt-Shapira Y, Perl O, Ravia A, Amir D, Eisen A, Bezalel $\mathrm{V}$, et al. Altered responses to social chemosignals in autism spectrum disorder. Nat Neurosci. 2018;21:111-9.

39. McAlonan GM, Daly E, Kumari V, Critchley HD, van AT, Suckling J, et al. Brain anatomy and sensorimotor gating in Asperger's syndrome. Brain. 2002;125:1594-606.

40. Belmonte MK, Cook EH Jr., Anderson GM, Rubenstein JL, Greenough WT, Beckel-Mitchener A, et al. Autism as a disorder of neural information processing: directions for research and targets for therapy. Mol Psychiatry. 2004;9:646-63.

41. Perry W, Minassian A, Lopez B, Maron L, Lincoln A. Sensorimotor gating deficits in adults with autism. Biol Psychiatry. 2007;61:482-6.

42. Yuhas J, Cordeiro L, Tassone F, Ballinger E, Schneider A, Long JM, et al. Brief report: sensorimotor gating in idiopathic autism and autism associated with fragile $\mathrm{X}$ syndrome. J Autism Dev Disord. 2010;41:248-53.

43. Ornitz EM, Lane SJ, Sugiyama T, de Traversay J. Startle modulation studies in autism. J Autism Dev Disord. 1993;23:619-37.

44. Girirajan S, Rosenfeld JA, Coe BP, Parikh S, Friedman N, Goldstein A, et al. Phenotypic heterogeneity of genomic disorders and rare copy-number variants. N Engl J Med. 2012;367:1321-31.

45. Niemi MEK, Martin HC, Rice DL, Gallone G, Gordon S, Kelemen $\mathrm{M}$, et al. Common genetic variants contribute to risk of rare severe neurodevelopmental disorders. Nature. 2018;562:268-71.

46. Hudac CM, Bove J, Barber S, Duyzend M, Wallace A, Martin CL, et al. Evaluating heterogeneity in ASD symptomatology, cognitive ability, and adaptive functioning among 16p11.2 CNV carriers. Autism Res. 2020. https://doi.org/10.1002/aur.2332.

47. Sandhu KV, Lang D, Muller B, Nullmeier S, Yanagawa Y, Schwegler H, et al. Glutamic acid decarboxylase 67 haplodeficiency impairs social behavior in mice. Genes Brain Behav. 2014;13:439-50.

48. Han S, Tai C, Westenbroek RE, Yu FH, Cheah CS, Potter GB, et al. Autistic-like behaviour in Scn1a+/- mice and rescue by enhanced GABA-mediated neurotransmission. Nature. 2012;489: 385-90.

49. Leekam SR, Nieto C, Libby SJ, Wing L, Gould J. Describing the sensory abnormalities of children and adults with autism. J Autism Dev Disord. 2007;37:894-910.
50. Portmann T, Yang M, Mao R, Panagiotakos G, Ellegood J, Dolen $\mathrm{G}$, et al. Behavioral abnormalities and circuit defects in the basal ganglia of a mouse model of $16 \mathrm{p} 11.2$ deletion syndrome. Cell Rep. 2014;7:1077-92.

51. Yang M, Mahrt EJ, Lewis F, Foley G, Portmann T, Dolmetsch $\mathrm{RE}$, et al. 16p11.2 Deletion syndrome mice display sensory and ultrasonic vocalization deficits during social interactions. Autism Res. 2015;8:507-21.

52. Yang M, Lewis FC, Sarvi MS, Foley GM, Crawley JN. 16p11.2 deletion mice display cognitive deficits in touchscreen learning and novelty recognition tasks. Learn Mem. 2015;22:622-32.

53. Valor LM, Grant SG. Clustered gene expression changes flank targeted gene loci in knockout mice. PLoS ONE. 2007;2:e1303.

54. Yang S, Farias M, Kapfhamer D, Tobias J, Grant G, Abel T, et al. Biochemical, molecular and behavioral phenotypes of Rab3A mutations in the mouse. Genes Brain Behav. 2007;6:77-96.

55. Ricard G, Molina J, Chrast J, Gu W, Gheldof N, Pradervand S, et al. Phenotypic consequences of copy number variation: insights from Smith-Magenis and Potocki-Lupski syndrome mouse models. PLoS Biol. 2010;8:e1000543.

56. O'Leary J, Osborne LR. Global analysis of gene expression in the developing brain of Gtf2ird1 knockout mice. PLoS ONE. 2011;6: e23868.

57. Hiroi N, Hiramoto T, Harper KM, Suzuki G, Boku S. Mouse models of 22q11.2-associated autism spectrum disorder. Autism. 2012;:S1:1-9.

58. Ozonoff S, Young GS, Belding A, Hill M, Hill A, Hutman T, et al. The broader autism phenotype in infancy: when does it emerge? J Am Acad Child Adolesc Psychiatry. 2014;53: 398-407.

59. Ozonoff S, Young GS, Brian J, Charman T, Shephard E, Solish A, et al. Diagnosis of autism spectrum disorder after age 5 in children evaluated longitudinally since infancy. J Am Acad Child Adolesc Psychiatry. 2018;57:849-57 e842.

60. Estes A, Zwaigenbaum L, Gu H, St John T, Paterson S, Elison JT, et al. Behavioral, cognitive, and adaptive development in infants with autism spectrum disorder in the first 2 years of life. J Neurodev Disord. 2015;7:24.

61. Zwaigenbaum L, Bryson S, Garon N. Early identification of autism spectrum disorders. Behav Brain Res. 2013;251:133-46.

\section{Affiliations}

Mitsuteru Nakamura $\mathbb{1}^{1,2} \cdot$ Kenny $\mathrm{Ye}^{3} \cdot$ Mariel Barbachan e Silva ${ }^{4} \cdot$ Takahira Yamauchi $^{1} \cdot$ Daniel J. Hoeppner $\left(\mathbb{1}^{5}\right.$. Amir Fayyazuddin $\circledast^{5}$. Gina Kang ${ }^{1}$. Emi A. Yuda ${ }^{6}$. Masako Nagashima ${ }^{7}$. Shingo Enomoto ${ }^{7}$. Takeshi Hiramoto ${ }^{1}$. Richard Sharp ${ }^{8}$ - Itaru Kaneko $\mathbb{D}^{9} \cdot$ Katsunori Tajinda $^{5} \cdot$ Megumi Adachi $^{5} \cdot$ Takuma Mihara $^{10}$ - Shinichi Tokuno ${ }^{2,11}$. Mark A. Geyer ${ }^{8}$ Pilib Ó Broin ${ }^{4}$ Mitsuyuki Matsumoto ${ }^{5,10} \cdot$ Noboru Hiroi $\mathbb{C}^{1,12,13,14}$

1 Department of Pharmacology, University of Texas Health Science Center at San Antonio, San Antonio, TX, USA

2 Department of Bioengineering, Graduate School of Engineering, The University of Tokyo, Tokyo, Japan

3 Department of Epidemiology and Health Science, Albert Einstein College of Medicine, Bronx, NY, USA

4 School of Mathematics, Statistics \& Applied Mathematics, National University of Ireland Galway, Galway, Ireland

5 La Jolla Laboratory, Astellas Research Institute of America LLC, San Diego, CA, USA
6 Graduate School of Engineering, Tohoku University, Sendai, Japan

7 Department of Psychiatry, Albert Einstein College of Medicine, Bronx, NY, USA

8 Department of Psychiatry, University of California, San Diego, La Jolla, CA, USA

9 Graduate School of Medical Sciences, Nagoya City University, Nagoya, Japan

10 Drug Discovery Research, Astellas Pharma Inc., Ibaraki, Japan 
11 Graduate School of Health Innovation, Kanagawa University of Human Services, Kawasaki-shi, Kanagawa, Japan

12 Department of Cellular and Integrative Physiology, University of Texas Health Science Center at San Antonio, San Antonio, TX, USA
13 Department of Cell Systems Anatomy, University of Texas Health Science Center at San Antonio, San Antonio, TX, USA

14 Department of Psychiatry, University of Texas Health Science Center at San Antonio, San Antonio, TX, USA 